Bol. Acad. peru. leng. 62.2017 (123-144)

\title{
Si fuera tolteca: César Moro y las redes intelectuales mexicanas en los años cuarenta
}

\section{If he were Toltec: Cesar Moro and the Mexican intellectual networks in the 1940s}

\author{
YANNA HADATTY MORA \\ Universidad Nacional Autónoma de México
}

Resumen:

Este artículo busca vincular a Moro con el campo cultural mexicano, poniendo énfasis en su vinculación con diferentes grupos políticos y estéticos durante su llamado «período mexicano» (1938-1948). Habla de su conexión con los exiliados en México, antes y después de la ruptura con el movimiento surrealista. Al mismo tiempo, presenta el análisis de un poema poco atendido, «Sueño de un dependiente de barbería a las tres de la tarde». Plantea que se trata de un poema de este período, y no de los años previos a México. Analiza la significación de la cultura tolteca en términos culturales de la época, es decir, para la intelectualidad mexicana de los años cuarenta. Propone la importancia de leerlo dentro de estas coordenadas.

Abstract:

This article aims to connect Moro to the Mexican cultural sphere, placing special emphasis on his link 
with different aesthetic and political groups during his so-called "Mexican period" (1938-1948). It mainly covers his connection with the exiles in Mexico, before and after the breakdown with the surrealist movement. At the same time, it presents a poem analysis which receives little attention, "Sueño de un dependiente de barbería a las tres de la tarde". ('Dream of a barber shop's dependent at three in the afternoon'). This poem is considered to belong to this period, and not to the previous years before Mexico. This paper also analyses the meaning of the Toltec culture in cultural terms of the epoch, i.e., for the Mexican intelligentsia of the 1940s. It proposes the importance of reading Moro under these perspective.

Palabras clave: surrealismo, Contemporáneos, antifascismo, Dyn y Wolfgang Paalen.

Keywords: Surrealism, contemporary, anti-fascism, Dyn and Wolfgang Paalen.

Recibido: 25/10/2017

Aceptado: 15/12/2017

Mucha tinta ha corrido respecto a César Moro y su obra, el acento puesto sobre todo en torno a la estética surrealista de su poesía y de su obra plástica, a su labor de traductor y de crítico de arte, a su autodefinición como exiliado sin importar el país de residencia. Esta comunicación no quiere apartarse del todo de estos tópicos, aunque quizás sí altere su recorrido: busca revisar las redes intelectuales en las que 
participó Moro durante sus años mexicanos, para arriesgar luego una posible interpretación de un verso incluido en uno de sus poemas.

Como es bien conocido, México resulta determinante en la carrera de Moro: durante su década mexicana (marzo de 1938-abril de 1948) ${ }^{1}$ publica dos de sus tres poemarios, ambos en francés; recupera el español para escribir los poemas del que se constituiría en su libro póstumo, La tortuga ecuestre; pinta, expone, se vincula de manera militante con varias asociaciones artísticas y culturales. Vale la pena recordarlas a vuelo de pájaro.

En primer lugar, con el grupo de exiliados españoles y franceses, y sus proyectos políticos y artísticos, al trabajar en la librería Quetzal entre 1939 y 1945. En segundo, con el surrealismo internacional, al organizar la Exposición Internacional Surrealista junto con Wolfgang Paalen y André Breton en la Galería de Arte Mexicano, a fines de 1939, que se exhibe durante enero y febrero de 1940. En tercer lugar, con lo que podríamos denominar como el postsurrealismo pictórico-literario, al patrocinar y colaborar con la revista Dyn, que se publica en México, aunque en francés e inglés únicamente, entre 1942 y 1945, movida por el temperamento y la militancia del austriaco Wolfgang Paalen, que justamente en sus páginas hace una recusación pública del surrealismo. En cuarto lugar, con un cenáculo que podríamos simplificar grandemente al aglutinarlo

\footnotetext{
1 Para medir la importancia del período mexicano, lapso en que se concentra este artículo, valga resaltar que coincide por completo con el contenido del tomo II de la Obra poética completa de César Moro, editada por Ricardo Silva-Santisteban en el 2016 como Clásicos Peruanos 8, por la Academia Peruana de la Lengua y la Librería Anticuaria Sur. Es decir, que quienes estudian su obra lo ubican como su segundo período lírico, dentro de tres en total.
} 
como el circuito de los Contemporáneos, mediado por el contacto entrañable con el pintor Agustín Lazo y el poeta Xavier Villaurrutia, en la medida en que el autor peruano contribuye con una de sus publicaciones periódicas, El Hijo Pródigo, entre 1943 a 1945.

El singular entretejido de las redes intelectuales de la época, determinado en los círculos intelectuales y artísticos de esos años en mayor grado por la coincidencia política, el exilio y el internacionalismo, que por la plena coincidencia estética, explica que un espacio como la librería Quetzal funcionara por ejemplo también como lugar de consignación de dibujos de Leonora Carrington, Wolfgang Paalen, Esteban Francés, Agustín Lazo y Remedios Varo, así como del propio Moro, con lo que todos los círculos terminan unidos. Pero no únicamente los une el antifascismo; una figura de conexión entre Quetzal y el grupo de los Contemporáneos, por ejemplo, sería Eduardo Villaseñor -importante político, diplomático y economista mexicano, escritor cercano al grupo- propulsor, junto con Daniel Cosío Villegas, de la creación del Fondo de Cultura Económica, y uno de los intermediarios del financiamiento de ediciones Quetzal.

\section{Exilio español, resistencia francesa: librería Quetzal}

La librería Quetzal es el espacio de difusión de la editorial del mismo nombre (Quetzal S. A.) en su segunda época. La editorial, refundada por Julián Gorkin, Michel Berveiller y Bartolomé Costa-Amic, se adscribe a la trayectoria de sus dueños, como parte de un núcleo republicano catalán de escritores, editores y libreros, o bien 
de militantes antifascistas franceses. Quetzal había sido fundada originalmente por Ramón Sender, y vendida en 1941 a este trío, cuando el dueño original acepta una plaza universitaria en California. Las ediciones bilingües francés-español constituían la línea fuerte de Quetzal,

lo singular de Quetzal en esos años es su dedicación a dobles ediciones (en lengua original y en traducción al español) de libros importantes de la literatura francesa con los que se pretendía, por un lado, divulgar en México la mejor literatura francesa, y por el otro suplir el parón de la edición en Francia como consecuencia de la Ocupación alemana (Negritas y Cursivas. Libros e Historia Editorial 2014).

La editorial dura hasta 1945, año en que se funda el sello Costa-Amic ${ }^{2}$. Según referencias biográficas, Moro trabajó en la librería Quetzal a partir de 1941, situada en el pasaje Iturbide $18^{3}$, verdadero espacio de sociabilización de los artistas e intelectuales de izquierda de la época. Su perfil engarzaría sin disonancias en un circuito antifranquista, antifascista, antihitleriano y antiestalinista que propugna la mencionada editorial en sus catálogos: durante esos años Quetzal publica libros como Páginas del destierro, de Álvaro Albornoz;

2 Este trayecto se encuentra bien estudiado como parte de las editoriales del exilio español y catalán en México desde hace muchos años. Quetzal resulta una más de las empresas de prestigio editorial y fracaso económico en que el exiliado catalán participa: «Costa-Amic [...] escribió libros, participó en numerosas empresas editoriales junto a republicanos españoles y mexicanos, aventuras de tanto prestigio cultural como pésimos resultados económicos (Ediciones Libres, Publicaciones Panamericanas, Ediciones Quetzal, etc.) e inició en México un sistema de edición mancomunada que permitió a muchos escritores noveles mexicanos ver publicada su primera obra» (Férriz 2002: 235-236).

3 Esta información aparece en una publicidad de la revista Arquitectura y lo demás. No 1, mayo de 1945, pág. 72. 
Refugiados a través de Europa, Hombres contra Hitler, de Fritz Max Cahen (traducido por Concha de Albornoz); El oscuro invasor, de Franz von Rintelen; Advertencia a Francia, de Paul Reynaud; Mission ou dimission de la France, de Jules Romains; Hitler contra Stalin, de Victor Serge; Caníbales políticos: Hitler y Stalin en España, de Julián Gorkin.

Habría que recordar en este contexto el móvil político que determina el exilio del peruano: en Lima Emilio Adolfo Westphalen fue apresado y a Moro se le desterró a México, luego de que les requisaron todos los ejemplares de la revista clandestina $C A D R E$, que publicaban ambos, órgano de la asociación que respondía a la misma sigla, Comité de Amigos de los Defensores de la República Española, durante la dictadura de Óscar R. Benavides, en 1937. Es decir, que al verse forzado al exilio por su adhesión a la causa republicana, coincide también con los fundadores de Quetzal y la propuesta editorial que ellos amparan. En palabras de Fabienne Bradu, César Moro «atendía los clientes en la librería». Lo que es sabido, es que - como en otras publicaciones de la época- los colaboradores aprovechaban para publicitar su obra.

El mismo Moro y Benjamin Péret aprovechaban ese espacio para tomar suscripciones de sus poemarios (Bradu 1997: 45). Un importante capital francés sostiene la empresa hasta 1946.

\section{Exposición Internacional del Surrealismo: Wolfgang Paalen, André Breton, César Moro}

La historia del surrealismo en México mucho debe a la estadía de César Moro en esas tierras. Como afirma 
José Ricardo Hernández Echávarri en su tesis doctoral sobre Moro en México, allí «asume el papel de un destacado animador del movimiento surrealista que buscaba renovarse y encontrar en [...] tierras [mexicanas] un nuevo imaginario de lo maravilloso» (2011: 27). Moro «escribe reseñas, asiste a sus clases de pintura en [la Escuela de Artes Plásticas de] La Esmeralda, entra en contacto con escritores importantes de México y con la comunidad de artistas europeos [...] casi todos exiliados en la diáspora de la Segunda Guerra» (Hernández 2011: 27).

Probablemente su primera actividad local de difusión del surrealismo sea la aparición de una antología llamada La poesía surrealista, ideada, traducida y prologada por el peruano, tan pronto llegó, pues aparece en mayo de 1938. Circuló como suplemento de la revista Poesía, empresa de breve duración conducida por Neftalí Beltrán. En lo que se designa como «una noticia de César Moro» aparece una presentación que es más bien un manifiesto:

El surrealismo es el cordón que une la bomba de dinamita con el fuego para hacer volar la montaña. La cita de las tormentas portadoras del rayo y de la lluvia de fuego. El bosque virgen y la miríada de aves de plumaje eléctrico cubriendo el cielo tempestuoso. [...] Los nombres de Sade, Lautreamont, Rimbaud, Jarry, en formas diversas y delirantes de aerolito sobre una sábana de sangre transparente que agita el viento nocturno sobre el basalto ardiente del insomnio (Moro 1938: 6).

En su labor de traductor, da a conocer en otras dos publicaciones (El Hijo Pródigo, Letras de México) a los surrealistas franceses, nómina en la cual gracias a Moro 
destacan los menos usuales Giorgio de Chirico y Pablo Picasso, junto con el repertorio usual: Breton, Arp, Eluard, Dalí, Peret.

Si bien el texto de presentación de Moro a la Exposición Internacional Surrealista es muy conocido, vale la pena citarlo para recordar nuevamente su colindancia con el manifiesto, pues en su mejor tradición inicia justamente con tono epifánico, anunciando el advenimiento de la nueva era:

El siglo XIX estalla en una granada fantástica, se abre en un sostenido fuego de artificio, el árbol de la sangre al desnudo, en un cráter manando maravillas hacia 1910, fecha histórica, en la que Pablo Picasso, el incomparable, inicia su búsqueda designada con el impropio nombre de cubismo. El milagro comenzado entonces [...] (Moro 1940: 1).

Más adelante se reafirma la identidad del país donde se realiza la exhibición, repitiendo el tópico de la vinculación de México como patria del surrealismo, situando los referentes no en un presente histórico sino en un tiempo mítico, que recoge también una construcción ancestral mitificada:

Por primera vez en México, desde siglos, asistimos a la combustión del cielo, mil signos se confunden y se distinguen en la conjugación de constelaciones que reanudan la brillante noche precolombina. La Noche Purísima del Nuevo Continente en que grandiosas fuerzas de sueño entrechocaban las formidables mandíbulas de la civilización en México y de la civilización en el Perú. Países que guardan, a pesar de la invasión de los bárbaros españoles y de las secuelas que aún persisten, millares de puntos luminosos que deben sumarse bien pronto a la línea de fuego del surrealismo internacional (Moro 1940: 2). 
Llama la atención la inusual nómina de los pintores mexicanos dentro de la selección surrealista, quizá vinculada a la referida red intelectual de Moro, que permite que aparezcan obras de amigos que no comparten cabalmente dicha estética: exhiben en esa ocasión Agustín Lazo y Xavier Villaurrutia, pero también Manuel Rodríguez Lozano, Roberto Montenegro, Guillermo Meza, Antonio Ruiz «el Corcito», y los exiliados Carlos Mérida y José Moreno Villa, guatemalteco y español respectivamente. Es preciso subrayar que estos aparecen como nómina aparte, al final del catálogo, agrupados como «Pintores mexicanos». Dentro del listado en orden alfabético de artistas surrealistas, sin embargo, se incluye a tres nacionales: Frida Kahlo y Diego Rivera - cuya presencia se debe con seguridad más a André Breton que a Moro, pues el último en más de una ocasión objetará vincularlos con el movimiento surrealista-, así como el fotógrafo Manuel Álvarez Bravo —una de cuyas obras, «Parábola óptica», es justamente la imagen de portada del catálogo de la Exposición-, cuya inclusión resulta más inobjetable y cercana a los gustos de Moro ${ }^{4}$.

\section{Dyn (1942-1944): postsurrealismo. Wolfgang Paalen, Alice Rahon}

Dyn ha sido considerada una de las revistas más maravillosas dedicada al arte moderno (Kloyber 2000). Se publica en la Ciudad de México durante la II Guerra Mundial. La dirige el austriaco Wolfgang Paalen. Paalen y Alice Rahon, su esposa en aquel entonces, llegan por

4 Y más persistente: en 1943 reseñará el peruano el libro Escultura azteca. 20 fotos de Manuel Álvarez Bravo para El Hijo Pródigo. 
invitación de Frida Kahlo. Después de la exposición de 1940, Paalen termina su relación con el surrealismo, e inicia la apuesta por un arte que fuera síntesis de la ciencia moderna, el arte moderno y el arte nativo de América.

Esta voluntad de incluir en sus intereses el arte nativo es sumamente notoria, porque coexisten intereses etnológicos - que hacen, por ejemplo, que la pareja viaje, junto con la fotógrafa Eva Sulzer, a Alaska para documentar y estudiar las culturas totémicas vivas-. Y que el más importante número de la revista (el doble 4-5) se dedique al arte americano. Según la historiadora del arte mexicano Lourdes Andrade, existe distancia entre publicaciones internacionales surrealistas como $V V V$ o The Surreal Revolution por la obsesión de Paalen - era la revista de un hombre solo, con contribuciones de sus amigos - por la etnografía, el arte contemporáneo y la poesía. Representa un espacio de doble exilio: de los países de origen, y del surrealismo. Los tópicos mexicanos son la herencia indígena, tema sobre el que publican Miguel Covarrubias y Alfonso Caso. Y el arte contemporáneo mexicano, donde aparecen las fotografías de Manuel Álvarez Bravo y la obra de Carlos Mérida, naturalizado mexicano para entonces. Andrade insiste en que, de los surrealistas asentados en México, Paalen es el más fascinado con el país.

En cuanto al contenido, Dyn presenta un material riquísimo. Por una parte, la escisión de surrealismo y estalinismo puede documentarse allí, en un manifiesto «por la moralidad objetiva» firmado por Moro, ambos Paalen, Sulzer, Charles Givors y John Dawson. Por otra parte, el conocido adiós al surrealismo de Paalen. 
Pero también el interés genuino por las excavaciones arqueológicas en proceso, como documenta el texto de Covarrubias sobre Tlatilco, o la inclusión de un poema («Xiuhtecuhtli») de los Cantares mexicanos. Y las fotos de Martín Chambi de Machu Picchu.

\section{Dice Hernández Echávarri:}

El elemento más destacable de Moro, en relación con el surrealismo, es la conciencia de una «americanización» del movimiento, al que busca incorporar mitos y símbolos prehispánicos (como en su artículo Coricancha). En cierto sentido podríamos decir que llevó a cabo una tarea paralela a la de varios pintores (como Wifredo Lam). En sentido literal y metafórico, Moro es el traductor del surrealismo para la cultura americana. Sin haber escrito un poema significativo de gran aliento en esta línea de fusión entre los elementos surrealistas y los símbolos prehispánicos, la discusión abierta por Moro y su propia práctica como poeta (aun cuando hayan tardado décadas en conocerse) permitieron y fomentaron la creación de grandes poemas que unen la escritura surrealista y la simbología prehispánica [...] Asimismo, sin el ejemplo de Moro es inconcebible la obra de ciertos poetas posteriores que ahondan en las posibilidades del surrealismo como parte constitutiva de la tradición hispanoamericana (2011: 3).

\section{Contemporáneos: Xavier Villaurrutia y Agustín Lazo, El Hijo Pródigo}

Al recuperar la historia del surrealismo en México, Luis Mario Schneider recuerda los nexos que posibilitaron la llegada de Moro:

Llegó a México, incitado por el México de Agustín Lazo, a quien conocía cuando el pintor, escenógrafo y dramaturgo estudiaba en el atelier de Charles Dullin en la 
Francia de finales de la década del 20. Vino por Agustín Lazo, y aquí heredó también a su mejor amigo: Xavier Villaurrutia. Cuando los surrealistas -Benjamin Péret, Remedios Varo, Wolfgang Paalen, Alice Rahon, Leonora Carrington- llegaron después de 1940 terminó de completar ese círculo inquietante y exclusivo del mundo de Moro en nuestro país (Schneider 2010: 3).

La relación con Villaurrutia no pasa sin embargo por la coincidencia en el surrealismo. El grupo de los Contemporáneos, literatos emblemáticos que son bautizados así por la historiografía literaria, tardíamente a raíz de la publicación de la revista del mismo nombre, es reacio a las vanguardias, si bien presenta un gran conocimiento de textos que llega a divulgar en sus revistas, como es el caso de la escritura de Joyce. Se trata - especialmente en el caso de Villaurrutia- de autores modernos sin demasiada violencia rupturista, con raíces en la renovación poética y dramatúrgica de André Gide, Jean Giraudoux, Jean Genet. El aprecio de Villaurrutia por Moro, según el periodista mexicano Rafael Vargas, no se da por la poesía o el arte surrealistas, pues el mexicano será siempre impermeable ante el surrealismo. Pero sí de empatía en las lecturas, así Villaurrutia le abre las puertas para publicar en las revistas El Hijo Pródigo y Letras de México. Es famosa una carta que le dirige complacido por su obra, ya de regreso en Lima. Emilio Adolfo Westphalen participará también de esta amistad.

Querido Xavier, gracias por tu libro, por tu país, realidades latinoamericanas. Perdón si no supe expresar nuestra cabal admiración; tú sabes leer entre líneas. Que la vida — la admirable, la pavorosa vida- continúe desenvolviendo sus hilos; amar es al fin una indolencia. 
¿Cómo no seguir en los sitios de peligro, donde no caben ni salvación ni regreso?

Tanto peor si la realidad vence una vez y otra y convence a los eternos convencidos trayendo entre los brazos verdaderos despojos: el hierro y el cemento o la hoz y el martillo como argumentos definitivos para justificar la prodigiosa bestialización de la vida humana.

Ese mundo no es el nuestro (Moro 1991: 41).

La prematura muerte de Villaurrutia provoca uno de los momentos más tristes en la vida de Moro, que siempre reconoció generosamente su talento, y se identificó con él. Moro se vincula también con otros autores afines al grupo, como Elías Nandino o Eduardo Luquín.

\section{5. «Si fuera tolteca...»}

Como es sabido, los toltecas eran un grupo migrante prehispánico, inicialmente nómada, pero que finalmente se establece en la ciudad de Tollan, su centro urbano más importante, cuyo auge se sitúa entre los años 900 y 1200 d. C. De ellos procede el culto a Quetzalcoatl, que presentará gran arraigo en el territorio mesoamericano. Su documento de referencia antiguo más conocido es la denominada Historia tolteca-chichimeca, códice mixto del siglo XVI. En cuanto a la vigencia del término para Moro, las excavaciones en Tula se llevaron a cabo justamente cuando el poeta peruano se encontraba en México, en la década de los cuarenta, a cargo del antropólogo Jorge R. Acosta. Tula había sido reconocida como importante sitio arqueológico desde el XIX, pero la excavación data de entonces, cuando se encuentra el templo de Tlahuizcalpantecuhtli, señor de la Aurora, deidad del amanecer, 
vinculada al culto a Venus. La divulgación empieza de inmediato, el mismo Acosta publica en la Revista Mexicana de Estudios Antropológicos el artículo «Exploraciones en Tula, Hidalgo, 1940». Un año después se realiza la primera mesa redonda sobre «Tula y los toltecas», en donde los hallazgos antropológicos, junto con estudios etnohistóricos, llevan a establecer que la mitológica Tollan no es Teotihuacan sino Tula, Hidalgo. Entre 1937 y 1947 aparecen de Enrique Juan Palacios, Arqueología de México: culturas arcaica y tolteca como volumen primero de una Miscelánea Antropológica (México, Imprenta Mundial, 1937, volumen 4 de una Enciclopedia Ilustrada Antropológica); en Copenhague, la edición facsimilar de Ernst Mengin de la referida Historia tolteca-chichimeca en náhuatl (E. Munsksgaard, 1942, Historia tolteca-chichimeca Liber in lingua Nahuatl manuscriptus picturisque ornatus, ut est conservatus in Bibliotheca Nationis Gallicae Parisiensi sub numeris XLVI-LVIIIbis cum praefatione in lingua Britannica, Gallica, Germanica et Hispana atque indice paginarum edidit Ernst Mengin); y la misma Historia tolteca-chichimeca en edición mexicana, subtitulada Anales de Quauhtinchan (México, Librería Robredo, 1947, versión preparada y anotada por Heinrich Berlin, en colaboración con Silvia Rendón).

Por su parte, para aproximarnos al sentido metafórico del término, la revista Cemento de arquitectura y construcción que se publica entre 1925 y 1930, es relevada por la publicación hermana Tolteca (1929-1932), ambas a cargo del publicista Federico Sánchez Fogarty, uno de los fundadores del Comité para Propagar el Uso del Cemento Portland, que circulaba con alrededor de diez mil ejemplares, y que pasó de promover el material 
(cemento armado) a la marca nacional (la Tolteca, si bien establecida con capital inglés), vinculada tanto con las publicaciones norteamericanas modernas de la construcción como con las europeas. La vinculación de los toltecas como civilizadores y grandes constructores queda reafirmada así en el siglo XX mexicano por el uso del término por parte de una empresa que se dedica a importar y luego fabricar el cemento tipo portland, la Tolteca.

«Sueño de un dependiente de barbería a las tres de la tarde» ve la luz en 1976, cuando aparece la antología $L a$ tortuga ecuestre y otros textos, publicada póstumamente por Julio Ortega en la colección Altazor, bajo el sello de Monte Ávila Editores en Caracas, al retrabajar para un público más amplio la antología de Moro de 1969 hecha junto con André Coyné. Si bien la reciente edición de la obra poética completa lo ubica en un período previo (tomo I, pp. 76-80), es decir, entre los poemas que se habrían escrito entre 1924 y 1938 en el derrotero LimaParís-Lima. Por los siguientes elementos que expongo, considero que se trata con seguridad de un poema de César Moro correspondiente al período mexicano.

Ortega lo presenta en el apartado segundo, «Otros poemas en español (1927-1949)», seguramente porque fijarle una fecha exacta resulta imposible. Insisto en que se puede ceñir sin embargo el período, afirmando que se trata de un poema de la etapa mexicana de Moro: los referentes a otomíes y toltecas así lo indicarían, más aún la mención del tango El choclo, pues tienta pensar que Moro alude al tango tras popularizarse en 1947 una versión del mismo que debe su letra a Enrique Santos Discépolo; y, como puede rastrearse, el mencionado 
tango aparece cantado por Libertad Lamarque en la película mexicana de Luis Buñuel Gran casino ${ }^{5}$.

Se trata de un poema construido en primera persona, cuyo tema es el tedio ante la hora muerta en las actividades y la ansiedad del anhelo de un encuentro con el objeto amoroso, poco proclive a realizarse, elípticamente resumido: «Nada [...] / es incomprensible / si supieras decirme la hora del día / de un encuentro casual».

Dividido en tres partes desiguales, de 81, 10 y 21 versos respectivamente, una estrofa a la mitad del primer fragmento llama especialmente nuestra atención y motiva esta lectura:

Si fuera tolteca dirían

Éste es un tolteca de primera

Debió de haber nacido a mediados de mayo

$\mathrm{O}$ en algún otro mes

Según el calendario más o menos antiguo [...]

(Moro 1976: 54)

La construcción en modo potencial o condicional, lingüísticamente perteneciente al tipo condicional de lo «poco probable de ocurrir», se establece a partir de entonces como dinámica poética en una deriva lógica de la advertencia mencionada al inicio [ «Nada... es incomprensible / si supieras decirme la hora del día / de un encuentro casual»], y persiste en estrofas

5 Una historia muy singular tiene este tango, con música y letra originales de Ángel Villoldo y sucesivas letras de Juan Carlos Maraldo Catán y Enrique Santos Discépolo. Véase «El tango el choclo y sus letras», http:/ / www.terapiatanguera. com.ar/Grandes\%20Tangos/manus_el_choclo.htm. Las distintas letras pueden escucharse en el sitio http:/ / www.todotango.com/musica/tema/24/El-choclo/. 
sucesivas hasta cerrar el primer fragmento: «si fuera tolteca», "si fuera chino», "si fuera sirio-libanés», y, aumentando la libertad asociativa, «si fuera caballo de carrera», "si fuera una cantante de ópera», "si fuera carbonero», "si fuera farmacéutico», «si fuera un coche de caballos», "si fuera un tigre» (Moro 1976: 54-55). El tono que predomina en este ejercicio de divagación lúdica, como resulta manifiesto, es el de la ocurrencia. Como en un juego verbal, mientras más ilógicos los conectivos, más lograda la estrofa, en una estrategia propia del juego infantil antes que del surrealismo. Citamos para ejemplificar: «Si fuera una cantante de ópera tendría ocho cines privados», "si fuera carbonero / tendría un palacio de diamantes en una playa de cartón», «Si fuera tigre querría ser un kiosko de periódicos / [...] o una botella de limonada» (Moro 1976: 55).

Toda la cohesión del poema radica en este divagar ante la hora vacía, que va cambiando de tono. El segundo fragmento es una divagación completa en torno no a un condicional, sino a un pasado onírico: «En Alaska era un globo aerostático / teñido de azul cubierto de martas». Una estampa del espacio de nieve eterna que concluye «Ni un solo recuerdo sobre la blancura / una esperanza apenas de algo negro ${ }^{6}$ (Moro 1976: 55-56). La monotonía en extremo del blanco de la nieve de Alaska, permite la transferencia de sentidos entre el tiempo sin eventos del presente del poema, la barbería a las tres de la tarde, sin clientes, pero sobre

6 Seguramente hay un juego de referentes al mencionar Alaska al viaje de Westphalen, Rahon y Sulzer. Cada vez que se menciona el tópico, Moro menciona de manera expresa al primero en este sentido. 
todo sin la visita anhelada, y el paisaje polar sin un solo recuerdo por el blanco continuo.

La tercera parte del poema sufre un giro, y se vuelve una pesadilla que se anuncia apenas en un par de versos iniciales que trazan ya un paisaje apocalíptico: «Cuando la mañana salió era un pedrusco / en un país vecino al salir de la guerra», para detenerse luego en una evocación de memorias aciagas, ominosas: "Volvió entonces el recuerdo / De un columpio caldeado / Que pretendía ahorcar a un niño», o bien de la evocación de los momentos y referentes compartidos que ya no están: "Del tango "el Choclo" / De "Eugenia Grandet" / De los cuentos de Calleja empastados / De un agua furiosa que me envolvía para aplacarme asfixiándome / Todos los recuerdos amargos de la infancia / Se agolparon como en un ventisquero».

Es necesario en este punto recordar el aserto de Yolanda Westphalen al concluir su pionero estudio sobre Moro, que el poeta «representante de la marginalidad y la oposición a toda forma de oficialismo» se revela interconectado según su análisis no con los mitos en torno al Titicaca sino con Pachacamac, asociado con el Auca y la marginalidad (2011: 132). Probablemente algo semejante ocurra con Moro en este poema, cuando decide poner en el centro el sugerente «Si fuera tolteca» - no azteca, no maya, sino tolteca-. Es decir, y en el imaginario de su época, que se identifica utópicamente como parte de una cultura ancilar tenida por sabia, religiosa, civilizatoria, capaz de permitir la convivencia de pueblos plurilingües y pluriétnicos en sus territorios, cultura constructora; y no con la conquistadora, monolingüe, imperialista que representa lo teotihuacano. 
Sintetizo que lo que dice el poema en esta lectura podría resumirse en «si fuera mexicano, quisiera ser tolteca», como "quisiera ser Contemporáneo», como «quisiera ser Dyn», y asumo todo el riesgo interpretativo, al proponer que resulta la imagen más cabal que tenemos de Moro en su participación del campo cultural mexicano, cercano solo a quienes se encuentran lejos del oficialismo y del centro, es decir, a aquellos artistas e intelectuales exiliados de la resistencia antifascista, del surrealismo y postsurrealismo, o bien a autores mexicanos no canónicos, como Xavier Villaurrutia y Agustín Lazo (en su doble condición de marginalidad en tanto homosexuales, y en tanto no pertenecientes a una estética nacionalista). La única banda que permitiría que entre risas le colocaran sus amigos sería quizás esa, «tolteca de primera». 


\section{Bibliografía}

BRADU, Fabienne. «Bartomeu Costa-Amic», en Vuelta. No 253. México, diciembre de 1997, págs. 41-45.

ESTELA, Carlos y José Ignacio PADILLA (eds.). Amour à Moro. Homenaje a César Moro. Lima, Signo Lotófago, 2003.

FÉRRIZ ROURE, Teresa. «Bartomeu Costa-Amic, in memoriam (1911-2002)», en Migraciones \& Exilios: Cuadernos de la Asociación para el estudio de los exilios y migraciones ibéricos contemporáneos. № 3. 2002, págs. 235-236.

GALLO, Rubén. «Chapter 4. Cement», en Mexican Modernity, The Avant-Garde and the Technological Revolution. Cambridge, Massachusetts Institute of Technology, 2005, págs. 168-198.

GALLO, Rubén e Ignacio PADILLA. Heterodoxos mexicanos. Una antología dialogada. Colección 2 en Fondo. México, Fondo de Cultura Económica, 2006.

GREET, Michele. «César Moro's Transnational Surrealism», en Journal of Surrealism and the Americas. Vol. 7. No 1. 2013, págs. 19-51.

HERNÁNDEZ ECHÁVARRI, José Ricardo. César Moro en México: los versos de un voluntario inadaptado. Tesis doctoral en Literatura Hispánica. México, Colegio de México, 2011.

KLOYBER, Christian. Wolfgang Paalen's DYN. The Complete Reprint. Viena, VBK, Springer Verlag, 2000.

LEIDENBERGER, Georg. «Tres revistas mexicanas de arquitectura. Portavoces de la modernidad: 1923-1950», en Anales del Instituto de Investigaciones Estéticas. Vol. 34. № 101. México, noviembre de 2012, págs. 109-138. 
MORO, César. La tortuga ecuestre y otros textos. Edición de Julio Ortega. Colección Altazor. Caracas, Monte Ávila, 1976.

. «Carta a Xavier Villaurrutia», en Biblioteca de México. № 1. Enero-febrero de 1991, págs. 40-41.

. Obra poética completa I y II. Edición, traducción y cronología de Ricardo Silva-Santisteban. Lima, Academia Peruana de la Lengua, Sur Librería Anticuaria, 2016.

MUÑOZ CARRASCO, Olga. «Vivencias peruanas: el exilio y la Guerra Civil española», en Revista de Filología Románica. Anejo VII, 2011, págs. 279-287.

NEGRITAS Y CURSIVAS. LIBROS E HISTORIA EDITORIAL. «Libros en francés en México, la segunda etapa de Quetzal», 21 de noviembre de 2014, https://negritasycursivas.wordpress.com/2014/11/21/libros-en-frances-en-mexico-la-segunda-etapa-de-quetzal/ (revisión 15 de septiembre, 2016).

NICHOLSON, Melanie. «César Moro: Exile and the Poetic Imagination», en Revista Hispánica Moderna. Vol. 68. № 1. Junio de 2015, págs. 39-57.

ROSSELL, Cecilia. «Estilo y estructura en la Historia tolteca-chichimeca», en Desacatos. Revista de Antropología Social. No 22, septiembre/diciembre de 2006, págs. 65-92.

RUIZ AYALA, Iván. César Moro y la tortuga ecuestre (dos lecturas). Prólogo de André Coyné. Lima, Banco Central de Reserva del Perú, 1998.

SCHNEIDER, Luis Mario. "Nota introductoria», en César $\mathrm{MORO}$, Los surrealistas franceses. México, Universidad Nacional Autónoma de México (Coordinación de Difusión Cultural / Dirección de Literatura), 2010. 
VARGAS, Rafael. «Apuntes sobre la amistad César Moro y Villaurrutia», en Biblioteca de México. Homenaje a Xavier Villaurrutia. No 64, julio-agosto de 2001, págs. 17-19.

. «César Moro bajo el cielo de México», en Nexos. 27 de abril de 2016, pp.

WESTPHALEN, Yolanda. César Moro: la poética del ritual y la escritura mítica de la modernidad. Lima, Fondo Editorial de la Universidad Nacional Mayor de San Marcos, 2001.

WESTPHALEN, Yolanda (comp.). César Moro y el surrealismo en América Latina. Lima, Centro Cultural de España/Fondo Editorial de la Universidad Nacional Mayor de San Marcos, 2005. 